\title{
Estrategias de autorregulación para la habilidad de escritura en inglés: Un proyecto de aula en primaria
}

\section{Self-regulation strategies and writing skills in english: A classroom project of primary school}

\author{
Mena Montoya Andrés Felipe ${ }^{1}$ \\ Zapata Cano Lina Janeth ${ }^{2}$ \\ Castro-Camelo Adriana ${ }^{3}$ \\ Conejo Carrasco Francisco ${ }^{4}$
}

\section{RESUMEN}

Este articulo surge como una reflexión de la investigación "Estrategias de autorregulación en la habilidad de escritura en inglés: Un proyecto de aula para mejorar el desempeño de los estudiantes de grado quinto de la Institución Educativa Fe y alegría de Bello Antioquia", escrita en el entorno de la Maestría en Educación Virtual

1 Magíster en Comunicación-Educación en la línea Medios Interactivos de la Universidad Distrital Francisco José de Caldas y Licenciada en Lenguas Modernas de la misma universidad. Docente-investigadora con experiencia en el desarrollo de proyectos pedagógicos, comunicativos y educomunicativos. Correo electrónico: acastrocame@uniminuto.edu.co

2 Candidato de maestría en educación Corporación Universitaria Minuto de Dios y Licenciado en inglés de la Universidad Católica Luis Amigó. Correo electrónico: andres.mena@uniminuto.edu.co

$3 \quad$ Candidata de maestría en educación Corporación Universitaria Minuto de Dios y Licenciado en inglés de la Universidad Católica Luis Amigó. Correo electrónico: lina. zapata@uniminuto.edu.co

4. Coordinador del área transversal de Formación Humana en la Corporación Universitaria Minuto de Dios. https://orcid.org/0000-0002-3490-1937. Correo electrónico: jcquiroz11@hotmail.com. y a Distancia de la Corporación Universitaria Minuto de Dios. El objetivo de este estudio fue analizar las estrategias de autorregulación que inciden en el aprendizaje de los estudiantes del grado quinto de la institución frente al desempeño de las habilidades de escritura en inglés. Para esto, se realizaron entrevistas individuales a los estudiantes de grado quinto con el objeto de indagar sobre los pasos implementados a la hora de construir sus producciones escritas en inglés y los hábitos de estudio utilizados a la hora de desarrollar dichas actividades. Así mismo, se implementó un análisis documental con la idea de establecer nexos entre el saber de los estudiantes y la forma en que adquieren nuevos conocimientos y evidenciar el control y el uso de estrategias de aprendizaje en cada uno de los 
tasks ${ }^{4}$ de la clase de inglés. Esta investigación se desarrolló bajo el enfoque cualitativo, con un alcance descriptivo. Como instrumentos de recolección de datos se utilizaron las entrevistas a los estudiantes y los análisis de la información. Estos permitieron el posterior análisis de los diferentes instrumentos de recolección de datos.

Como principales hallazgos, este estudio revela que los estudiantes apoyan sus textos de la clase de inglés en la visión que les proporciona un revisor como un adulto, un tutor o sus padres de familia debido a que esto les da la seguridad sobre el trabajo realizado; así mismo, para que los estudiantes generen actitudes que permitan mejorar la autorregulación en su proceso, es imperativo establecer hábitos de estudio. Entre los encontrados en este estudio, se evidencia que los estudiantes prefieren realizar los ejercicios propuestos en ambientes de estudios silenciosos y tranquilos, ya que así se optimizan sus resultados académicos.

\section{PALABRAS CLAVE}

Autorregulación- habilidad escrita- motivaciónestrategias de aprendizaje- tasks.

\section{ABSTRACT}

This article arises as a reflection of the research named "self-regulation strategies in English writing: A classroom project to improve the fifth grader students' performance of Institución educative Fe y Alegría of Bello Antioquia." Written in the environment of the Virtual and Distance Master's Degree in Education of Corporación Universitaria Minuto de Dios. The objective of this study was to analyze the self-regulation strategies that influences in the learning process of the fifth grade students of the public institution on the writing skills performance in English. For this, individual interviews were conducted to every single fifth grade student in order to inquire

4 Es la palabra en inglés que significa tarea. Este concepto es utilizado por la docente de inglés para nombrar a cada una de las actividades que constituyen las construcciones del saber. about the steps implemented at the moment of constructing the written productions in English, as so as the learning styles implemented to develop those activities. Likewise, a documentary analysis was implemented with the idea of establishing links between what the students know and the way in which they acquire a new knowledge. Furthermore, to evidence the usage and control of learning strategies in every single tasks from the English class. This research was developed under the qualitative approach based on the inductive method. As information synthesization method, excel and google forms were used. Those tools let the later analysis of the different data collection instruments.

Like main findings, this study reveals that the students support their English class texts on the view that is provided by a reviewer like an adult, a tutor, or their parents. This, due to those reviewers give security on the work done. Furthermore, for the students to generate attitudes that allow to improve self-regulation in their process, it's mandatory to establish study habits. Among the found in this study, it is evidenced that students prefer to do exercises proposed in silent and quiet studying environments, since this way their academic results are optimized.

\section{KEY WORDS}

Self-regulation- writing skill-motivation-learning strategies-tasks.

\section{INTRODUCCIÓN}

En una época donde los ritmos de aprendizaje de los estudiantes han cambiado debido a los transformaciones generacionales, la introducción de la tecnología y los intereses de los jóvenes, se hace necesario buscar nuevas formas de enseñanza encaminados a mejorar los procesos de aprendizaje de los estudiantes. Es por esto, que la autorregulación resulta ser una herramienta útil, ya que, esto posibilita que los estudiantes adquieran habilidades 
pensando en sus propios intereses y ritmos de aprendizaje.

El Marco Común Europeo de Referencia es parte fundamental para delimitar el currículo de los estudiantes del sistema educativo colombiano, ya que los Estándares Básicos en Lengua Extranjeras y los Derechos Básicos de Aprendizaje se basan en estos principios para enmarcar las metas de aprendizaje de los estudiantes. Es por esto que, basándose en el establecimiento de $\mathrm{A} 2$ básico como marco de medida, se evidencia en la población a intervenir que los estudiantes no alcanzan el nivel deseado, de acuerdo con los exámenes diagnósticos realizados en los momentos de clase.

A pesar del esfuerzo y reconocimiento de la relevancia de la autonomía en el proceso educativo por parte de los docentes, no es muy visible o evidente, la forma cómo se pretende aplicar en el aula de clase. Más allá de nombrar e invitar a un estudio autónomo, el cuestionamiento iría en dirección a cómo hacer posible que el aula de clase sea lo suficientemente motivadora para que sea el impulso a generar esta conducta.

Por ende, entre su propuesta de formación, considera que, en referencia al aprendizaje de inglés, trabajar por ellos mismos, auto corregirse y corregir a los demás, es pilar de mayor necesidad en el ámbito de aprendizaje de lengua extranjera. Entender en este caso, que cada individuo debe comprender sus diferentes formas de trabajar, sus referencias, roles en el proceso y descubrimiento de la forma en la que cada uno aprende, que varía de los demás; por esto, el maestro sería un tutor que guía, para que cada uno particularice su aprendizaje.

Acorde a estos principios de contexto, SáizManzanares \& Pérez-Pérez(2016) realizaron una investigación donde se pretendía evaluar cómo impacta el entrenamiento de la autorregulación en el desempeño académico. Estos autores afirman que los aprendizajes son más profundos y significativos en la medida que la intervención modifica en cierto sentido, la forma como se implementan las estrategias de autorregulación. los mismso autores concluyeron que realizar un entrenamiento en estrategias de autorregulación facilita la formación de un aprendizaje más reflexivo.

Dicho esto, Torrano, Fuentes \& Soria (2017) descubren que existen una serie de estrategias didácticas en la instrucción docente; por ejemplo (Enseñanza directa de estrategias, modelado, práctica guiada y autonomía, autoobservación y práctica auto-reflexiva), todas estas, pensadas para desarrollar habilidades autorreguladas en los estudiantes. Sin embargo, existe un reto grande a la hora de adquirir un aprendizaje autorregulado, y este radica en desarrollar investigaciones sobre la integración de estrategias de autorregulación en diversas asignaturas escolares (Torrano, Fuentes y Soria, 2017). De acuerdo con Schunk y Zimmerman citado en Torrano, Fuentes y Soria (2017), "es importante incorporar las estrategias y enseñar a los alumnos a valorarlas, modificarlas y adaptarlas ante distintas situaciones de aprendizaje". (p.29)

En investigaciones recientes sobre estrategias de aprendizaje, (Pinto, Martínez y Taracido, 2016) reconocen que los estudiantes hábiles para la comprensión lectora utilizan estrategias de aprendizaje. Los estudios de Sainz y Pérez (2016) indagan sobre la autorregulación y la mejora del autoconocimiento en resolución de problemas. Sus hallazgos muestran que aumentar la utilización de estrategias metacognitivas de autoconocimiento y planificación en los educandos de bachillerato después de entrenarlos en metacognición autorregulada aplicado en resolución de problemas mejora el empleo de estrategias para codificar la información. 
Después de las consideraciones anteriores, en esta investigación se analizan las estrategias de autorregulación que inciden en el aprendizaje de los estudiantes del grado quinto de una institución pública frente al desempeño de las habilidades de escritura en inglés. En particular, aquellas estrategias que los estudiantes de manera inconsciente utilizan a la hora de desarrollar sus tasks de la clase de inglés. El interés de analizar las estrategias de autorregulación surge como necesidad de promover los principios institucionales de la autonomía y la autorregulación como estrategias para generar mejores competencias académicas, específicamente en la clase de inglés.

\section{METODOLOGÍA}

Esta investigación es de enfoque cualitativo a través de la Investigación - Acción, cuyo alcance es explicativo, ya que lo que se busca con este estudio es comprender y resolver problemáticas relacionadas con la habilidad escritural en inglés de los estudiantes del grado quinto a partir de la implementación de estrategias de regulación del aprendizaje.

En el desarrollo de esta investigación, se enfoca la atención en estudiantes que se encuentran entre los 9 y los 11 años de edad, en el grado de quinto de primaria de la Institución Educativa $\mathrm{Fe}$ y Alegría, los cuales pertenecen a un estrato socioeconómico 1 y 2 . Las técnicas de recolección de datos que se implementaron durante el proceso de investigación fueron; primero, el análisis de la información cuyo objetivo es la "captación, evaluación, selección y síntesis de los mensajes subyacentes en el contenido de los documentos, a partir del análisis de sus significados, a la luz de un problema determinado." (Dulzaides y Molina, 2004, p. 2) Este instrumento, permite a los investigadores el análisis de las composiciones escritas de los estudiantes llamadas "construcciones del saber" mediante el uso de estrategias de autorregulación que puedan influir positivamente en los resultados académicos en cuanto a la habilidad escrita en inglés. Este instrumento resulta esencial en el análisis de las estrategias utilizadas por los estudiantes de la institución ya que contribuye a una interpretación proyectiva de los saberes y adquisición de nuevos conocimientos de los estudiantes.

Como segunda técnica de recolección de datos, se implementó la entrevista semiestructurada; esta permitió al grupo de investigadores obtener información relacionada sobre el uso de estrategias de aprendizaje encaminadas a la autoobservación en el grupo de estudiantes de los grados $5^{\circ}$ en la institución pública. Esto, con el fin de establecer las estrategias que el grupo de estudiantes utiliza para favorecer los procesos de escritura en una lengua extranjera (inglés). La entrevista permitió obtener más información con relación al uso de estrategias por ser instrumento que permitió realizar preguntas abiertas donde los sujetos pudieron dar más información con respecto a la categoría a analizar. De acuerdo con Janesick en Sampieri (2014) la entrevista "se define como una reunión para conversar e intercambiar información entre una persona (el entrevistador) y otra (el entrevistado) u otras (entrevistados). En el último caso podría ser tal vez una pareja o un grupo pequeño como una familia."(p.436)

\section{RESULTADOS Y ANÁLISIS}

De acuerdo con línea enmarcada de propuesta investigativa, y luego de aplicar los diferentes instrumentos de investigación, como análisis de información, análisis documental y entrevistas semi-estructuradas, considerados pertinentes para el campo de estudio; se analizan los trabajos de los estudiantes que objetivo en donde se pretendía identificar la forma en la que estos logran los trabajos escriturales del área de inglés, evaluando la forma en la que se hacía conexiones entre los saberes previos y nexos con los nuevos conocimientos en cada una de las tasks enmarcadas en los proyectos de aula. 
Todo esto encaminado reconocer cómo ellos tienen control de las estrategias en cada una de las tasks que implicaba escritura en inglés y el monitoreo que hacen de su propio proceso.

En el siguiente diagrama se observa las respuestas proporcionadas por los estudiantes sobre cómo completan tasks de escritura.

Tabla 1. Resolución de tasks de escritura

Recuento de Cuando no sabes el significado en espanol de una palabra ¿Que herramienta usas? (Diccionario, traductor, otro cuál?)

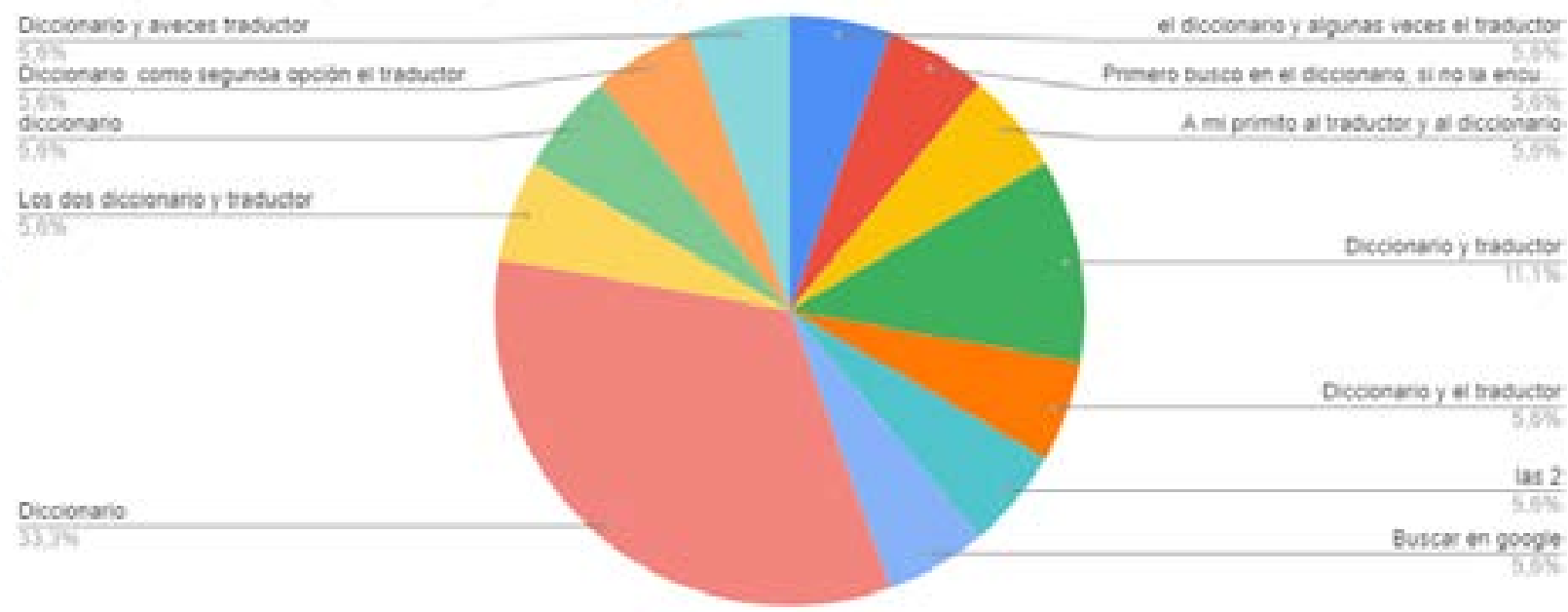

Nota: Elaboración fuente propia

Tabla 2. Uso de las TIC en el aprendizaje del inglés

\section{Recuento de ¿Tienes alguna página web que usas para estudiar inglés? si, Cual/ no}

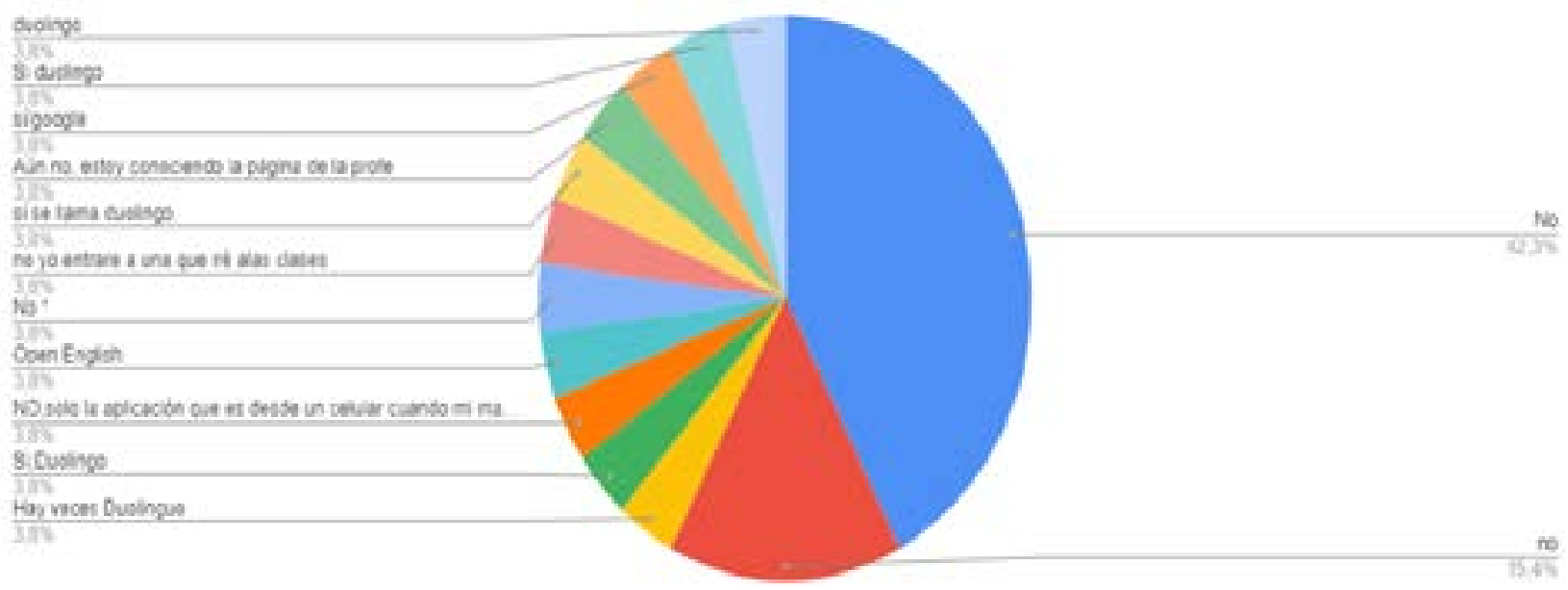

Nota: Elaboración fuente propia 
Resulta relevante identificar que como estrategia institucional de trabajo se encuentra la confrontación de escritura (Ver tabla 1). Esta es una forma de trabajar en donde la revisión y edición se hace imprescindible. Se realiza una primera versión de texto, supeditado a ser revisado por el docente, el padre de familia, un compañero de clase o quien se considere pertinente para cumplir este rol. Esta persona que revisa el texto hace observaciones sobre aspectos que pueden ser mejorados, que incluyen coherencia, cohesión, de carácter lingüístico o estilo; posteriormente se hace una retroalimentación con estos aspectos, donde el estudiante revisa estos apuntes y se hace las debidas modificaciones. Se ha observado que los estudiantes prefieren que este proceso sea realizado por el docente o el padre de familia acompañante.

De igual manera, es evidente lo descrito anteriormente en el documento como lo llaman en la institución educativa "Construcciones del saber" que son relatos individuales de carácter reflexivo en donde los estudiantes retoman asuntos relevantes del proyecto de aula y hablan de sus alcances, dificultades y nuevos saberes que se propiciaron en este sentido, siendo esto una muestra de que esta población reconoce en la escritura la forma de asentar ideas que propicien desarrollo de habilidades de pensamiento.

Se observa que la proporción de estudiantes que implementan esta estrategia e autorregulación es muy alta

Tabla 3. Correcciones post retroalimentación

\section{Cuando tienes errores en tus trabajos, ¿Haces correciones?}

\section{9 tespuestas}
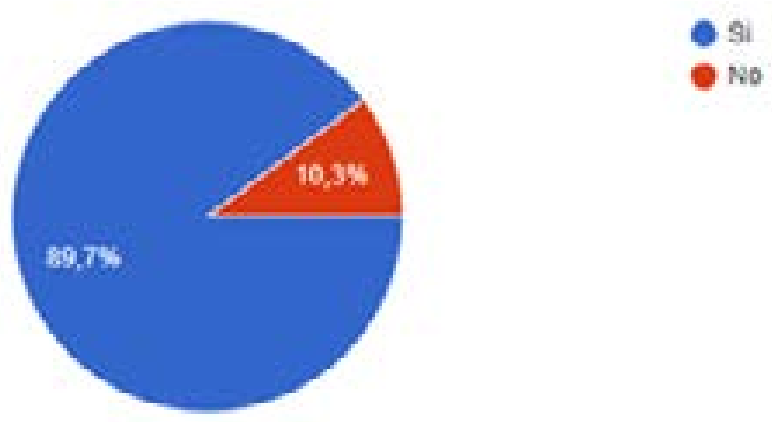

Nota: Elaboración fuente propia

En esta misma línea, en la implementación de una entrevista semiestructurada de carácter abierta, se pretendía describir los hábitos de estudio, los pasos o el proceso para hacer una composición escrita y evidenciar el uso de parte de los aprendices; por lo cual se usa como estrategia el análisis de datos cualitativos, Según Rodríguez, Gil y García en Rodríguez, Herrera y Lorenzo (2005) mencionan que "Este proceso quedaría configurado en torno a tres aspectos clave: reducción de datos, disposición y transformación de éstos y, por último, obtención de resultados y verificación de conclusiones." ( $p$. 5) ya que permite la comparación y análisis con un enfoque de investigación acción. En el enfoque de investigación-acción permitió según Sampieri (2014) “(...) la detección de necesidades (ya que ellos conocen mejor que nadie la problemática a resolver), el involucramiento con la estructura a modificar, el proceso a mejorar, las prácticas que requieren cambiarse y la implementación de los resultados del estudio" (p. 497) 
Obsérvese en el siguiente diagrama las estrategias implementadas por los estudiantes.

Tabla 4. Uso de actividades extra curriculares

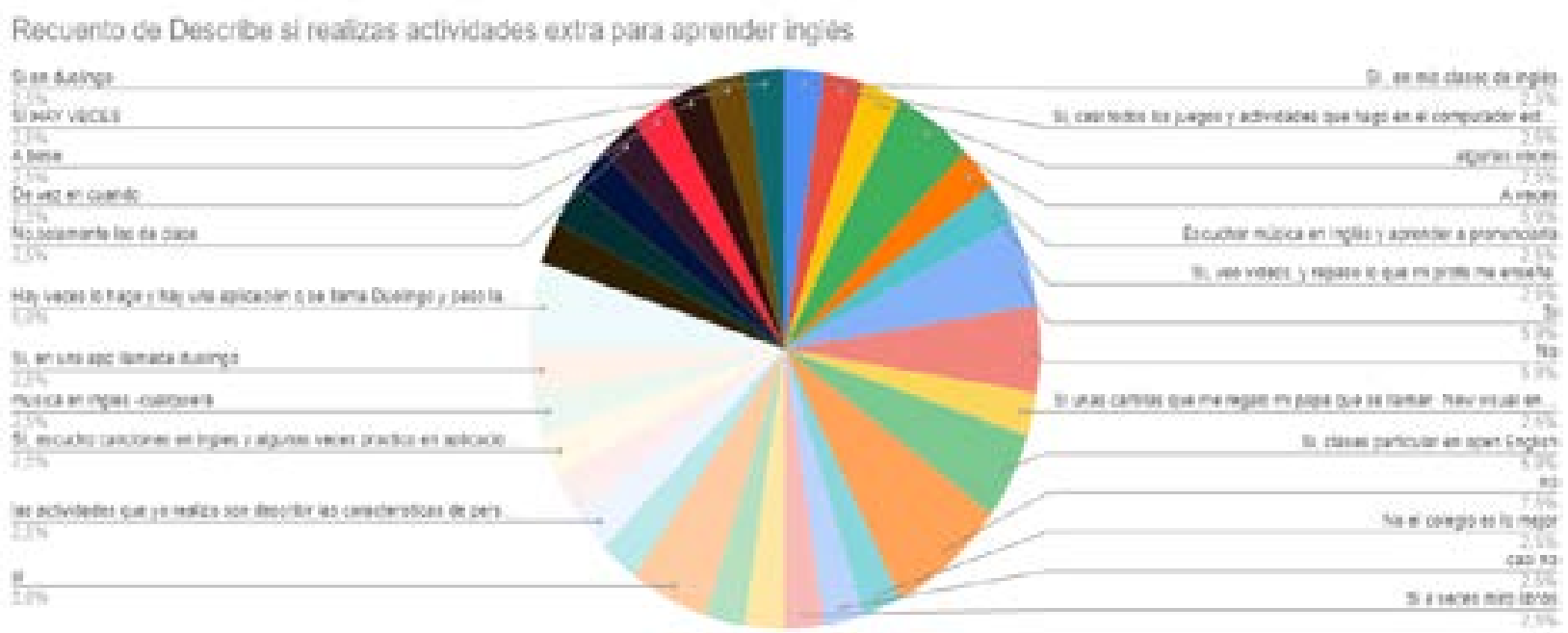

Nota: Elaboración fuente propia

Teniendo esto establecido, se analizan los resultados para poder establecer hallazgos contundentes en la población objeto de estudio. De acuerdo con lo analizado se detecta que gran parte de los estudiantes prefieren estrategias de monitoreo, es decir, en donde se encuentre un agente revisor de sus procesos que le reafirme cómo va su proceso. Para apoyar lingüísticamente la escritura apropiada, se evidencia la utilización de diccionario como herramienta de fundamentación léxica y el uso de dispositivos que les puedan ayudar a la revisión de textos o frases; evidenciando así características de la auto-observación de su proceso. Sin embargo, también se puede entender que no es parte habitual que los estudiantes usen pre-writing steps o etapas previas de escritura, sin embargo, la escritura, confrontación y revisión y es parte de sus hábitos.

Los métodos de estudio son formas en las que los aprendices identifican sus formas efectivas de estudiar, aprender y obtener conocimiento de acuerdo al propósito que tengan. El uso de internet y el traductor parecen ser los más comunes. Esto les ayuda también a asociar palabras que tengan alguna equivalencia en español, apoyándose así en su lengua materna (Hernández y García en Seder y Villalonga, 2016).

En esa misma línea, se considera la importancia de los hábitos de estudio en todo proceso de aprendizaje por lo que esta forma implica una maduración de las formas más apropiadas de obtención de conocimiento. Sin embargo, a pesar de reconocer esto, no se evidencia que los estudiantes tengan horarios, o disciplina $u$ organización de su tiempo, por lo que se realiza las sugerencias pertinentes a la institución para mejorar en este aspecto. Otro aspecto a resaltar es que los nexos de aprendizajes previos con los nuevos se hacen evidente cuando se encuentran tasks de escritura y usan estructuras gramaticales aprendidas previamente, ya sea con sustantivos, verbos y adjetivos de forma básica en donde se siguen modelos lingüísticos.

\section{CONCLUSIONES}

Con el devenir de los días, los aprendices de diferentes grados de escolaridad han 
reconocido la importancia de la autonomía y la autorregulación en sus procesos de aprendizaje. El desarrollo cognitvo y metacogtivo en la edad escolar temprana se alcanza de manera óptima generando competencias indispensables para la formación de los diferentes campos de vida de los aprendices, que van desde la resolución de problemas cotidianos hasta el ingenio para las actividades en áreas específicas. Cada vez más, las escuelas han incluido en sus currículos reflexiones y formas prácticas de implementar estas formas buscando que sus estudiantes desarrollen capacidades de formarse como seres autónomos cada vez a una edad más temprana. Aunque no es una tarea fácil, el fomento de la autorregulación en la escuela culmina en procesos formativos más conscientes que derivan en niveles de estudio y preparación para el campo científico e investigativo.

En este campo de desarrollo personal, los procesos educativos donde se procura por el pensamiento crítico, la creatividad y la autorregulación logran en el aprendiz equilibrio, en donde lo orientado por el docente se complementa en la comprensión de los procesos personales de aprendizaje y la operancia en la racionalización de los conceptos y la resolución de situaciones que se encuentra cada sujeto en campo académico, profesional y personal.

Ahora bien, el objetivo de la educación debe llevar a la singularidad personal, haciendo que los aprendices sean conscientes de sus fortalezas, debilidades, limitantes $y$ potencialidades. Comprendiendo que la relación constante con el mundo y la construcción del conocimiento responde a características individuales, se resalta que es relevante en este proceso construir hábitos de estudio y hacerlo consciente en la implementación de los mismos en su día a día, evaluando las estrategias que mejor se acomoden a sus necesidades, en aras de establecer valores de autoeficacia.

\section{REFERENCIAS BIBLIOGRÁFICAS}

Cuadros Luyo, V. C., Morán Pachas, B. V., \& Torres Jimenez, A. Y. (2017). Hábitos de estudio, disposición hacia el estudio y rendimiento académico en estudiantes de formación profesional técnica de un instituto armado.

Diego, M. C. G., Castañeda, E., \& Morales, J. M. M. (2018). Experiencia de innovación en el aula desde la autorregulación y los estilos de aprendizaje. Tendencias pedagógicas, (31), 137-148.

Dulzaides Iglesias, M. E., \& Molina Gómez, A. M. (2004). Análisis documental y de información: dos componentes de un mismo proceso. Acimed, 12(2), 1-1.

Hernández Sampieri, R., FERNÁNDEZ COLLADO, C., \& BAPTISTA LUCIO, P. (2014). Metodología de la Investigación. Sexta Edición. Mcgrawhill.

Pinto, N. S., Martínez, A. I. M., \& JiménezTaracido, L. (2016). Estrategias de aprendizaje, comprensión lectora y rendimiento académico en Educación Secundaria. Psicologia Escolar e Educacional, 20(3), 447-456.

Rodríguez Sabiote, C., Herrera Torres, L., \& Lorenzo Quiles, O. (2005). Teoría y práctica del análisis de datos cualitativos. Proceso general y criterios de calidad.

Román, Ó. C., \& Gaitero, Ó. G. (2017). El aprendizaje autorregulado y las estrategias de aprendizaje. Tendencias pedagógicas, (30), 117-130.

Sáiz-Manzanares, M. C., \& Pérez, M. I. P. (2016). Autorregulación y mejora 
del autoconocimiento en resolución de problemas. Psicología desde el Caribe, 33(1), 14-30.

Seder, A. C., \& Villalonga, H. B. (2016). Importancia de los hábitos de estudio en el rendimiento académico del adolescente: diferencias por género. Educatio Siglo XXI, 34(1 Marzo), 157-172.

Torrano, F., Fuentes, J. L., \& Soria, M. (2017). Aprendizaje autorregulado: estado de la cuestión y retos psicopedagógicos. Perfiles educativos, 39(156), 160-173. 